\title{
Estimate on the Dimension of Global Attractor for Nonlinear Higher-Order Coupled Kirchhoff Type Equations
}

\author{
Guoguang Lin, Lingjuan Hu \\ Department of Mathematics, Yunnan University, Kunming, China \\ Email: gglin@ynu.edu.cn, 879694199@qq.com
}

How to cite this paper: Lin, G.G. and Hu, L.J. (2018) Estimate on the Dimension of Global Attractor for Nonlinear HigherOrder Coupled Kirchhoff Type Equations. Advances in Pure Mathematics, 8, 11-24. https://doi.org/10.4236/apm.2018.81002

Received: December 7, 2017

Accepted: January 6, 2018

Published: January 9, 2018

Copyright $(9) 2018$ by authors and Scientific Research Publishing Inc. This work is licensed under the Creative Commons Attribution International License (CC BY 4.0).

http://creativecommons.org/licenses/by/4.0/

\begin{abstract}
In this paper, we investigate the finite dimensions of the global attractor for nonlinear higher-order coupled Kirchhoff type equations with strong linear damping in Hilbert spaces $E_{0}$ and $E_{1}$. Under the appropriate assumptions, we acquire a precise estimate of the upper bound for its Hausdorff and Fractal dimensions.
\end{abstract}

\section{Keywords}

Higher-Order, Kirchhoff-Type Equations, Global Attractor, Hausdorff Dimension, Fractal Dimension

\section{Introduction}

G. G. Lin and L. J., Hu have studied the existence of a global attractor for coupled Kirchhoff type equations with strongly linear damping in [1]. In this paper, we are concerned with the finite dimensions of the global attractor as mentioned above:

$$
\begin{gathered}
u_{t t}+M\left(\left\|D^{m} u\right\|^{2}+\left\|D^{m} v\right\|^{2}\right)(-\Delta)^{m} u+\beta(-\Delta)^{m} u_{t}+g_{1}(u, v) \\
=f_{1}(x), \text { in } \Omega \times[0,+\infty), \\
v_{t t}+M\left(\left\|D^{m} u\right\|^{2}+\left\|D^{m} v\right\|^{2}\right)(-\Delta)^{m} v+\beta(-\Delta)^{m} v_{t}+g_{2}(u, v) \\
=f_{2}(x), \text { in } \Omega \times[0,+\infty), \\
u(x, 0)=u_{0}(x), u_{t}(x, 0)=u_{1}(x), \quad x \in \Omega, \\
v(x, 0)=v_{0}(x), \quad v_{t}(x, 0)=v_{1}(x), \quad x \in \Omega,
\end{gathered}
$$




$$
\frac{\partial^{i} u}{\partial n^{i}}=0, \quad \frac{\partial^{i} v}{\partial n^{i}}=0, \quad i=0,1,2, \cdots, m-1, \quad x \in \partial \Omega, t \geq 0,
$$

where $\Omega$ is a bounded domain in $R^{n}$ with smooth boundary $\partial \Omega, \beta>0$ is real number, $m \geq 1$ is positive integer. $M(s)$ and $g_{j}(u, v)(j=1,2)$ are given functions later.

In demonstrating the longtime behavior of evolutional equation, we currently aim to show that the dynamics of the equation is finite dimensional. To be precise, one possible way to express it is to say that the dynamical systems of equation exists a global attractor with finite Hausdorff and Fractal dimensions.

Concerning the wave equation with linear and semi-linear dissipative system, existence of the global attractor with finite Hausdorff and Fractal dimensions is proved in [2], for the nonlinear wave equation, the existence of the global attractor with finite Hausdorff and Fractal dimensions is proved in [3] [4] [5]. When the equation is nonlinear, the process of dimension estimation is more complicated. The method of linearization works very well on it, and meanwhile we take fully consideration of assumptions on the nonlinearities of the equation.

Recently, Z. J, Yang [6] studied the longtime behavior of the Kirchhoff type equation with strong damping on $R^{N}$. It showed that the related continuous semi-group $S(t)$ possesses a global attractor which is connected and has finite Fractal and Hausdorff dimensions.

$$
u_{t t}-M\left(\|\nabla u\|^{2}\right) \Delta u-\Delta u_{t}+u+u_{t}+g(x, u)=f(x), \text { in } R^{N} \times R^{+} .
$$

At the same time, Z. J. Yang [7] dealt with the global attractors and their Hausdorff dimensions for a class of Kirchhoff models, and got the existence, regularity, and Hausdorff dimensions of global attractors for a class of Kirchhoff models arising in elastoplastic flow.

$$
u_{t t}-\operatorname{div}\left\{\sigma\left(|\nabla u|^{2}\right) \nabla u\right\}-\Delta u_{t}+\Delta^{2} u+h\left(u_{t}\right)+g(u)=f(x), \quad \text { in } \Omega \times R^{+} .
$$

Furthermore, X. M. Fan and S. F. Zhou [8] proved the existence of compact kernel sections for the process generated by strongly damped wave equations of non-degenerate Kirchhoff type modelling the nonlinear vibrations of an elastic string, and they obtained a precise estimate of upper bound of Hausdorff dimension of kernel sections.

$$
u_{t t}-\alpha \Delta u_{t}-\left(\beta+\gamma\left(\int_{\Omega}|\nabla u|^{2} \mathrm{~d} x\right)^{\rho}\right) \Delta u+h\left(u_{t}\right)+f(u, t)=g(x, t), \quad x \in \Omega, t>\tau .
$$

In addition, G. G. Lin and Y. L. Gao [9] studied the longtime behavior of solution to initial boundary value problem for a class of strongly damped higher-order Kirchhoff type equation:

$$
u_{t t}+(-\Delta)^{m} u_{t}+\left(\alpha+\beta\left\|\nabla^{m} u\right\|^{2}\right)^{q}(-\Delta)^{m} u+g(u)=f(x), \quad(x, t) \in \Omega \times[0,+\infty),
$$


they got the existence and uniqueness of the solution by the Galerkin method and obtained the existence of the global attractor in $H_{0}^{m}(\Omega) \times L^{2}(\Omega)$ according to the attractor theorem, besides, the estimation of the upper bound of Hausdorff dimension for the attractor was established.

The paper is arranged as follows. In Section 2, some preliminaries and main results are stated. In Section 3, in order to acquire the result of the estimation, we show the differentiability of semigroup. Eventually, the Hausdorff and Fractal dimensions of the global attractor for the dynamics system associated with problem (1.1)-(1.5) are discussed in detail.

\section{Preliminaries and Main Results}

Throughout this paper, we need some notations for convenience. We consider a family of Hilbert spaces $V_{a}=D\left(A^{a / 2}\right), a \in R$, whose inner products and norms are given by $(\cdot, \cdot)_{V_{a}}=\left(A^{a / 2}, A^{a / 2}\right)$ and $\|\cdot\|_{V_{a}}=\left\|A^{a / 2} \cdot\right\|$. Obviously

$$
V_{0}=L^{2}(\Omega), \quad V_{m}=H^{m} \times H_{0}^{1}, \quad V_{2 m}=H^{2 m} \times H_{0}^{1},
$$

we denote

$$
\begin{gathered}
E_{0}=V_{m} \times V_{0} \times V_{m} \times V_{0}, \\
E_{1}=V_{2 m} \times V_{m} \times V_{2 m} \times V_{m}, \\
E^{*}=V_{m} \times V_{m} .
\end{gathered}
$$

For our purpose, we define a weighted inner product and norm in $E_{0}$ by

$$
\begin{aligned}
& (\varphi, \tilde{\varphi})_{E_{0}}=\left(u_{1}, u_{2}\right)+\left(p_{1}, p_{2}\right)+\left(v_{1}, v_{2}\right)+\left(q_{1}, q_{2}\right), \\
& \|\varphi\|_{E_{0}}^{2}=(\varphi, \varphi)_{E_{0}}=\left\|A^{\frac{m}{2}} u_{1}\right\|^{2}+\left\|p_{1}\right\|^{2}+\left\|A^{\frac{m}{2}} v_{1}\right\|^{2}+\left\|q_{1}\right\|^{2},
\end{aligned}
$$

with any $\varphi=\left(u_{1}, p_{1}, v_{1}, q_{1}\right)^{\mathrm{T}}, \tilde{\varphi}=\left(u_{2}, p_{2}, v_{2}, q_{2}\right)^{\mathrm{T}} \in E_{0}$.

Next, we make the following assumptions for problem (1.1)-(1.5).

(A1) $M(s) \in C^{2}([0,+\infty), R)$ is not decreasing function and for positive constants $m_{0}, m_{1}$,

1) $0<\beta<m_{0} \leq M(s) \leq m_{1}$,

2) $m^{*}=\left\{\begin{array}{l}m_{0}, \frac{\mathrm{d}}{\mathrm{d} t}\left(\left\|D^{m} u\right\|^{2}+\left\|D^{m} v\right\|^{2}\right)>0 . \\ m_{1}, \frac{\mathrm{d}}{\mathrm{d} t}\left(\left\|D^{m} u\right\|^{2}+\left\|D^{m} v\right\|^{2}\right)<0 .\end{array}\right.$

(A2) There exists $0<\kappa_{1}, \kappa_{2}<1$, and for every $R_{0}$, there exist $c_{0}=c_{0}\left(R_{0}\right)$, $c_{0}^{\prime}=c_{0}^{\prime}\left(R_{0}\right)$ such that

$$
\begin{gathered}
\left\|g_{1 i}(\tilde{u}, \tilde{v})-g_{1 i}(u, v)\right\| \leq c_{0}\|(\tilde{u}, \tilde{v})-(u, v)\|_{E^{*}}^{\kappa_{1}}, \\
\left\|g_{2 i}(\tilde{u}, \tilde{v})-g_{2 i}(u, v)\right\| \leq c_{0}^{\prime}\|(\tilde{u}, \tilde{v})-(u, v)\|_{E^{*}}^{\alpha_{2}}, \quad(i=u, v), \\
\forall(\tilde{u}, \tilde{v}),(u, v) \in E^{*}, \quad\|(\tilde{u}, \tilde{v})\|_{E^{*}} \leq c\left(R_{0}\right),\|(u, v)\|_{E^{*}} \leq c\left(R_{0}\right) . \\
\text { where }\|(\tilde{u}, \tilde{v})-(u, v)\|_{E^{*}}^{\kappa}=\left\|D^{m}(\tilde{u}-u)\right\|^{\kappa}+\left\|D^{m}(\tilde{v}-v)\right\|^{\kappa} .
\end{gathered}
$$




\section{The Hausdorff and Fractal Dimensions of the Attractor}

In order to obtain the result of the dimension estimation, we should prepare the following lemmas.

Lemma 3.1. ([1]) Suppose that the assumptions of [1] hold, the constants $T>0, \beta>0$ and initial value $\left(u_{0}, p_{0}, v_{0}, q_{0}\right) \in E_{0}$, then for the problem (1.1)-(1.5), there exists a unique weak solution such that $(u, p, v, q) \in L^{\infty}\left((0,+\infty) ; E_{0}\right)$.

Lemma 3.2. ([1]) Suppose that $M(s)$ and $g_{i}(u, v)(i=1,2)$ satisfy assumptions of [1] respectively. Then for $\beta>0$, the problem (1.1)-(1.5) possesses the global attractor $A$, which is compact among bounded absorbing set $B_{0}$ in $E_{0}$, that is

$$
A=\omega\left(B_{0}\right)=\bigcap_{s \geq 0} \overline{\bigcup_{t \geq s} S(t) B_{0}},
$$

this lemma is result on the existence of a global attractor of system (1.1)-(1.5) generated by semigroup $S(t)$.

The first step will be to prove the differentiability of $S(t)$. Denote

$\bar{U}=(U, P, V, Q)$. In what follows, we put $M(s)=M\left(\left\|D^{m} u\right\|^{2}+\left\|D^{m} v\right\|^{2}\right)$ for simplicity. The first variation equations of (1.1)-(1.5) as the following

$$
\begin{gathered}
P_{t}+M(s) A^{m} U+2 M^{\prime}(s)\left(A^{\frac{m}{2}} U, A^{\frac{m}{2}} u\right) A^{m} u+2 M^{\prime}(s)\left(A^{\frac{m}{2}} V, A^{\frac{m}{2}} v\right) A^{m} u \\
-\varepsilon\left(\beta A^{m}-\varepsilon\right) U+\left(\beta A^{m}-\varepsilon\right) P+g_{1 u}(u, v) U+g_{1 v}(u, v) V=0, \\
Q_{t}+M(s) A^{m} V+2 M^{\prime}(s)\left(A^{\frac{m}{2}} U, A^{\frac{m}{2}} u\right) A^{m} v+2 M^{\prime}(s)\left(A^{\frac{m}{2}} V, A^{\frac{m}{2}} v\right) A^{m} v \\
-\varepsilon\left(\beta A^{m}-\varepsilon\right) V+\left(\beta A^{m}-\varepsilon\right) Q+g_{2 u}(u, v) U+g_{2 v}(u, v) V=0, \\
\left.(U, P, V, Q)\right|_{t=0}=(\xi, \zeta, \eta, \sigma), \\
\left.U(x, t)\right|_{x \in \partial \Omega}=\left.V(x, t)\right|_{x \in \partial \Omega}=0,
\end{gathered}
$$

where $U_{t}+\varepsilon U=P, V_{t}+\varepsilon V=Q,(\xi, \zeta, \eta, \sigma) \in E_{0}$, $(u, p, v, q)=S(t)\left(u_{0}, p_{0}, v_{0}, q_{0}\right)$ is the solution of Equations (1.1)-(1.5) with $\left(u_{0}, p_{0}, v_{0}, q_{0}\right) \in A$.

Given $\left(u_{0}, p_{0}, v_{0}, q_{0}\right) \in A$, the solution $S(t)\left(u_{0}, p_{0}, v_{0}, q_{0}\right) \in E_{0}$, by standard methods, we can prove that for any $(\xi, \zeta, \eta, \sigma) \in E_{0}$, the linear initial boundary value problem (3.1)-(3.4) possesses a unique solution $\bar{U}=(U(t), P(t), V(t), Q(t)) \in L^{\infty}\left(R, E_{0}\right)$.

Lemma 3.3. for any $t>0, R>0$, the mapping $S(t): E_{0} \rightarrow E_{0}$ is Frechet differentiable on $E_{0}$. Its differential at $\rho_{0}=\left(u_{0}, p_{0}, v_{0}, q_{0}\right)$ is the linear operator on $E_{0}$.

$$
D S(t) \rho_{0}:(\xi, \zeta, \eta, \sigma) \rightarrow(U, P, V, Q),
$$

where $\bar{U}=(U(t), P(t), V(t), Q(t))$ is the solution of (3.1)-(3.4).

Proof. Let $\rho_{0}=\left(u_{0}, p_{0}, v_{0}, q_{0}\right) \in E_{0}, \quad \tilde{\rho}_{0}=\left(u_{0}+\xi, p_{0}+\zeta, v_{0}+\eta, q_{0}+\sigma\right) \in E_{0}$ with $\left\|\rho_{0}\right\| \leq R_{0},\left\|\tilde{\rho}_{0}\right\| \leq R_{0}$, we denote $S(t) \rho_{0}=\rho=(u, p, v, q)$,

$S(t) \tilde{\rho}_{0}=\tilde{\rho}=(\tilde{u}, \tilde{p}, \tilde{v}, \tilde{q})$. First, we can prove a Lipschitz property of $S(t)$ on 
the bounded sets on $E_{0}$, that is

$$
\left\|S(t) \rho_{0}-S(t) \tilde{\rho}_{0}\right\|_{E_{0}}^{2} \leq \exp \left(c^{\prime} t\right)\|(\xi, \zeta, \eta, \sigma)\|_{E_{0}}^{2}, \forall t \geq 0 .
$$

We now consider the difference $\tilde{\rho}-\rho-\bar{U}=(\tilde{u}-u-U, \tilde{p}-p-P, \tilde{v}-v-V, \tilde{q}-q-Q)=(\omega, \theta, \gamma, \delta)$, with $\bar{U}=(U(t), P(t), V(t), Q(t))$ the solution of (3.1)-(3.4), clearly,

$$
\begin{aligned}
& \omega(0)=\theta(0)=\gamma(0)=\delta(0)=0 \\
& \theta_{t}-\varepsilon \theta+\beta A^{m} \theta+\varepsilon^{2} \omega-\beta \varepsilon A^{m} \omega+M(s) A^{m} w+g_{1 u}(u, v) \omega+g_{1 v}(u, v) \gamma \\
& =h_{1}+(M(s)-M(\tilde{s})) A^{m} \tilde{u}+2 M^{\prime}(s)\left(A^{\frac{m}{2}} U, A^{\frac{m}{2}} u\right) A^{m} u \\
& +2 M^{\prime}(s)\left(A^{\frac{m}{2}} V, A^{\frac{m}{2}} v\right) A^{m} u, \\
& \delta_{t}-\varepsilon \delta+\beta A^{m} \delta+\varepsilon^{2} \gamma-\beta \varepsilon A^{m} \gamma+M(s) A^{m} \gamma+g_{2 u}(u, v) \omega+g_{2 v}(u, v) \gamma \\
& =h_{2}+(M(s)-M(\tilde{s})) A^{m} \tilde{v}+2 M^{\prime}(s)\left(A^{\frac{m}{2}} U, A^{\frac{m}{2}} u\right) A^{m} v \\
& +2 M^{\prime}(s)\left(A^{\frac{m}{2}} V, A^{\frac{m}{2}} v\right) A^{m} v
\end{aligned}
$$

where $w_{t}+\varepsilon w=\theta, \gamma_{t}+\varepsilon \gamma=\delta$, with

$$
\begin{aligned}
& h_{1}=-\left(g_{1}(\tilde{u}, \tilde{v})-g_{1}(u, v)-g_{1 u}(u, v)(\tilde{u}-u)-g_{1 v}(u, v)(\tilde{v}-v)\right), \\
& h_{2}=-\left(g_{2}(\tilde{u}, \tilde{v})-g_{2}(u, v)-g_{2 u}(u, v)(\tilde{u}-u)-g_{2 v}(u, v)(\tilde{v}-v)\right) .
\end{aligned}
$$

We have

$$
\begin{aligned}
h_{1}= & -\int_{0}^{1}\left[\left\{g_{1 u}\left(u+\theta_{1}(\tilde{u}-u), v+\theta_{1}(\tilde{v}-v)\right)-g_{1 u}(u, v)\right\}(\tilde{u}-u)\right. \\
& \left.+\left\{g_{1 v}\left(u+\theta_{1}(\tilde{u}-u), v+\theta_{1}(\tilde{v}-v)\right)-g_{1 v}(u, v)\right\}(\tilde{v}-v)\right] \mathrm{d} \theta_{1}, \\
h_{2}= & -\int_{0}^{1}\left[\left\{g_{2 u}\left(u+\theta_{2}(\tilde{u}-u), v+\theta_{2}(\tilde{v}-v)\right)-g_{2 u}(u, v)\right\}(\tilde{u}-u)\right. \\
& \left.+\left\{g_{2 v}\left(u+\theta_{2}(\tilde{u}-u), v+\theta_{2}(\tilde{v}-v)\right)-g_{2 v}(u, v)\right\}(\tilde{v}-v)\right] \mathrm{d} \theta_{2},
\end{aligned}
$$

due to $\forall \theta_{1}, \theta_{2} \in[0,1]$,

$$
\begin{gathered}
\left\|g_{1 u}\left(u+\theta_{1}(\tilde{u}-u), v+\theta_{1}(\tilde{v}-v)\right)-g_{1 u}(u, v)\right\| \leq c_{0} \theta_{1}^{\kappa_{1}}\|(\tilde{u}, \tilde{v})-(u, v)\|_{E^{*}}^{\kappa_{1}}, \\
\left\|g_{1 v}\left(u+\theta_{1}(\tilde{u}-u), v+\theta_{1}(\tilde{v}-v)\right)-g_{1 v}(u, v)\right\| \leq c_{0} \theta_{1}^{\kappa_{1}}\|(\tilde{u}, \tilde{v})-(u, v)\|_{E^{*}}^{\kappa_{1}}, \\
\left\|g_{2 u}\left(u+\theta_{2}(\tilde{u}-u), v+\theta_{2}(\tilde{v}-v)\right)-g_{2 u}(u, v)\right\| \leq c_{0}^{\prime} \theta_{2}^{\kappa_{2}}\|(\tilde{u}, \tilde{v})-(u, v)\|_{E^{*}}^{\kappa_{2}}, \\
\left\|g_{2 v}\left(u+\theta_{2}(\tilde{u}-u), v+\theta_{2}(\tilde{v}-v)\right)-g_{2 v}(u, v)\right\| \leq c_{0}^{\prime} \theta_{2}^{\kappa_{2}}\|(\tilde{u}, \tilde{v})-(u, v)\|_{E^{*}}^{\kappa_{2}}, \\
\left\|h_{1}\right\| \leq 2 c_{1}\|(\tilde{u}, \tilde{v})-(u, v)\|_{E^{*}}^{\kappa_{1}+1} .
\end{gathered}
$$

Similarly

$$
\left\|h_{2}\right\| \leq 2 c_{1}^{\prime}\|(\tilde{u}, \tilde{v})-(u, v)\|_{E^{*}}^{K_{2}+1} .
$$


Taking the scalar product of each side of (3.8)-(3.9) with $\theta$ and $\delta$, and then we have

$$
\begin{aligned}
& \frac{1}{2} \frac{\mathrm{d}}{\mathrm{d} t}\left\{\|\theta\|^{2}+\|\delta\|^{2}+\varepsilon^{2}\left(\|\omega\|^{2}+\|\gamma\|^{2}\right)+\left(m^{*}-\beta \varepsilon\right)\left(\left\|A^{\frac{m}{2}} \omega\right\|^{2}+\left\|A^{\frac{m}{2}} \gamma\right\|^{2}\right)\right\} \\
& +\left(\frac{\beta}{2} \lambda_{1}^{m}-\varepsilon\right)\left(\|\theta\|^{2}+\|\delta\|^{2}\right)+\frac{\beta}{2}\left(\left\|A^{\frac{m}{2}} \theta\right\|^{2}+\left\|A^{\frac{m}{2}} \delta\right\|^{2}\right) \\
& +\varepsilon^{3}\left(\|\omega\|^{2}+\|\gamma\|^{2}\right)+\varepsilon\left(m_{0}-\beta \varepsilon\right)\left(\left\|A^{\frac{m}{2}} \omega\right\|^{2}+\left\|A^{\frac{m}{2}} \gamma\right\|^{2}\right) \\
& \leq\left(h_{1}-g_{1 u}(u, v) \omega-g_{1 v}(u, v) \gamma, \theta\right)+\left(h_{2}-g_{2 u}(u, v) \omega-g_{2 v}(u, v) \gamma, \delta\right) \\
& +\left((M(s)-M(\tilde{s})) A^{m} \tilde{u}, \theta\right)+\left((M(s)-M(\tilde{s})) A^{m} \tilde{v}, \delta\right) \\
& +\left(2 M^{\prime}(s)\left(A^{\frac{m}{2}} U, A^{\frac{m}{2}} u\right) A^{m} u+2 M^{\prime}(s)\left(A^{\frac{m}{2}} V, A^{\frac{m}{2}} v\right) A^{m} u, \theta\right) \\
& +\left(2 M^{\prime}(s)\left(A^{\frac{m}{2}} U, A^{\frac{m}{2}} u\right) A^{m} v+2 M^{\prime}(s)\left(A^{\frac{m}{2}} V, A^{\frac{m}{2}} v\right) A^{m} v, \delta\right) \\
& =\left(h_{1}-g_{1 u}(u, v) \omega-g_{1 v}(u, v) \gamma, \theta\right)+\left(h_{2}-g_{2 u}(u, v) \omega-g_{2 v}(u, v) \gamma, \delta\right) \\
& +\left(J_{1}, \theta\right)+\left(J_{1}^{\prime}, \delta\right) .
\end{aligned}
$$

Because of

$$
\begin{aligned}
& \left(h_{1}-g_{1 u}(u, v) \omega-g_{1 v}(u, v) \gamma, \theta\right) \\
\leq & 2 c_{1}\|\theta\|\|(\tilde{u}, \tilde{v})-(u, v)\|_{E^{*}}^{\kappa_{1}+1}+c_{2}\|\omega\|\|\theta\|+c_{3}\|\gamma\|\|\theta\|, \\
& \left(h_{2}-g_{2 u}(u, v) \omega-g_{2 v}(u, v) \gamma, \delta\right) \\
\leq & 2 c_{1}^{\prime}\|\delta\|(\tilde{u}, \tilde{v})-(u, v)\left\|_{E^{*}}^{\kappa_{2}+1}+c_{2}^{\prime}\right\| \omega\|\| \delta\left\|+c_{3}^{\prime}\right\| \gamma\|\| \delta \| . \\
J_{1}= & (M(s)-M(\tilde{s})) A^{m} \tilde{u}+2 M^{\prime}(s)\left(\left(A^{\frac{m}{2}} U, A^{\frac{m}{2}} u\right)+\left(A^{\frac{m}{2}} V, A^{\frac{m}{2}} v\right)\right) A^{m} u \\
=- & 2 M^{\prime}(s)\left(\left(-A^{\frac{m}{2}} U, A^{\frac{m}{2}} u\right)+\left(-A^{\frac{m}{2}} V, A^{\frac{m}{2}} v\right)\right) A^{m} u+M^{\prime}(\alpha s+(1-\alpha) \tilde{s}) \\
\times & {\left[\left(D^{m}(u-\tilde{u}), D^{m}(\tilde{u}+u)\right)+\left(D^{m}(v-\tilde{v}), D^{m}(\tilde{v}+v)\right)\right] A^{m} \tilde{u} } \\
= & \left(M^{\prime}(\alpha s+(1-\alpha) \tilde{s})-M^{\prime}(s)\right) \\
\times & {\left[\left(D^{m}(u-\tilde{u}), D^{m}(\tilde{u}+u)\right)+\left(D^{m}(v-\tilde{v}), D^{m}(\tilde{v}+v)\right)\right] A^{m} \tilde{u} } \\
+ & M^{\prime}(s)\left[\left(D^{m}(u-\tilde{u}), D^{m}(\tilde{u}+u)\right)+\left(D^{m}(v-\tilde{v}), D^{m}(\tilde{v}+v)\right)\right] A^{m} \tilde{u} \\
- & 2 M^{\prime}(s)\left[\left(D^{m}(u-\tilde{u}), D^{m} u\right)+\left(D^{m}(v-\tilde{v}), D^{m} v\right)\right] A^{m} \tilde{u} \\
+ & 2 M^{\prime}(s)\left[\left(D^{m}(u-\tilde{u}), D^{m} u\right)+\left(D^{m}(v-\tilde{v}), D^{m} v\right)\right] A^{m} \tilde{u} \\
- & 2 M^{\prime}(s)\left[\left(D^{m}(u-\tilde{u}), D^{m} u\right)+\left(D^{m}(v-\tilde{v}), D^{m} v\right)\right] A^{m} u \\
- & 2 M^{\prime}(s)\left(\left(D^{m} \omega, D^{m} u\right)+\left(D^{m} \gamma, D^{m} v\right)\right) A^{m} u
\end{aligned}
$$




$$
\begin{aligned}
\leq & M^{\prime \prime}(\xi)\left[\left(D^{m}(u-\tilde{u}), D^{m}(\tilde{u}+u)\right)+\left(D^{m}(v-\tilde{v}), D^{m}(\tilde{v}+v)\right)\right]^{2} A^{m} \tilde{u} \\
& +M^{\prime}(s)\left[\left(D^{m}(u-\tilde{u}), D^{m}(\tilde{u}-u)\right)+\left(D^{m}(v-\tilde{v}), D^{m}(\tilde{v}-v)\right)\right] A^{m} \tilde{u} \\
& +2 M^{\prime}(s)\left[\left(D^{m}(u-\tilde{u}), D^{m} u\right)+\left(D^{m}(v-\tilde{v}), D^{m} v\right)\right] A^{m}(\tilde{u}-u) \\
& -2 M^{\prime}(s)\left(\left(D^{m} \omega, D^{m} u\right)+\left(D^{m} \gamma, D^{m} v\right)\right) A^{m} u \\
= & I_{1}+I_{2}+I_{3}+I_{4} .
\end{aligned}
$$

Taking the scalar product of right side of (3.16) with $\theta$, and then we obtain $\left(I_{1}, \theta\right)$

$$
\begin{aligned}
& \leq\left(2 M^{\prime \prime}(\xi)\left[\left(D^{m}(u-\tilde{u}), D^{m}(\tilde{u}+u)\right)^{2}+\left(D^{m}(v-\tilde{v}), D^{m}(\tilde{v}+v)\right)^{2}\right] D^{m} \tilde{u}, D^{m} \theta\right) \\
& \leq 2 c_{4}\left(\frac{4}{\varepsilon^{2}}\left\|D^{m}(\tilde{u}-u)\right\|^{4}+\frac{4}{\varepsilon^{2}}\left\|D^{m}(\tilde{v}-v)\right\|^{4}+\frac{\varepsilon^{2}\left\|D^{m} \theta\right\|^{2}}{8}\right), \\
& \left(I_{2}, \theta\right) \\
& =\left(M^{\prime}(s)\left[\left(D^{m}(u-\tilde{u}), D^{m}(\tilde{u}-u)\right)+\left(D^{m}(v-\tilde{v}), D^{m}(\tilde{v}-v)\right)\right] D^{m} \tilde{u}, D^{m} \theta\right) \\
& \leq c_{5}\left(\frac{4}{\varepsilon^{2}}\left\|D^{m}(\tilde{u}-u)\right\|^{4}+\frac{4}{\varepsilon^{2}}\left\|D^{m}(\tilde{v}-v)\right\|^{4}+\frac{\varepsilon^{2}\left\|D^{m} \theta\right\|^{2}}{8}\right), \\
& \left(I_{3}, \theta\right) \leq\left(2 M^{\prime}(s)\left[\left(D^{m}(u-\tilde{u}), D^{m} u\right)+\left(D^{m}(v-\tilde{v}), D^{m} v\right)\right] D^{m}(\tilde{u}-u), D^{m} \theta\right) \\
& \leq 2 c_{6}\left(\frac{8}{\varepsilon^{2}}\left\|D^{m}(\tilde{u}-u)\right\|^{4}+\frac{4}{\varepsilon^{2}}\left\|D^{m}(\tilde{v}-v)\right\|^{4}+\frac{3 \varepsilon^{2}\left\|D^{m} \theta\right\|^{2}}{16}\right), \\
& \left(I_{4}, \theta\right)=\left(-2 M^{\prime}(s)\left[\left(D^{m} \omega, D^{m} u\right)+\left(D^{m} \gamma, D^{m} v\right)\right] D^{m} u, D^{m} \theta\right) \\
& \leq 2 c_{7}\left(\frac{4\left\|D^{m} \omega\right\|^{2}}{\varepsilon^{2}}+\frac{4\left\|D^{m} \gamma\right\|^{2}}{\varepsilon^{2}}+\frac{\varepsilon^{2}\left\|D^{m} \theta\right\|^{2}}{8}\right),
\end{aligned}
$$

which implies that

$\left|\left(J_{1}, \theta\right)\right| \leq \frac{C_{8}}{\varepsilon^{2}}\left(\left\|D^{m}(\tilde{u}-u)\right\|^{4}+\left\|D^{m}(\tilde{v}-v)\right\|^{4}+\left\|D^{m} \omega\right\|^{2}+\left\|D^{m} \gamma\right\|^{2}\right)+c_{9} \varepsilon^{2}\left\|D^{m} \theta\right\|^{2}$.

Analogously,

$$
\begin{aligned}
& \left(I_{1}^{\prime}, \delta\right) \leq 2 c_{4}^{\prime}\left(\frac{4}{\varepsilon^{2}}\left\|D^{m}(\tilde{u}-u)\right\|^{4}+\frac{4}{\varepsilon^{2}}\left\|D^{m}(\tilde{v}-v)\right\|^{4}+\frac{\varepsilon^{2}\left\|D^{m} \delta\right\|^{2}}{8}\right), \\
& \left(I_{2}^{\prime}, \delta\right) \leq c_{5}^{\prime}\left(\frac{4}{\varepsilon^{2}}\left\|D^{m}(\tilde{u}-u)\right\|^{4}+\frac{4}{\varepsilon^{2}}\left\|D^{m}(\tilde{v}-v)\right\|^{4}+\frac{\varepsilon^{2}\left\|D^{m} \delta\right\|^{2}}{8}\right) \\
& \left(I_{3}^{\prime}, \delta\right) \leq 2 c_{6}^{\prime}\left(\frac{4}{\varepsilon^{2}}\left\|D^{m}(\tilde{u}-u)\right\|^{4}+\frac{8}{\varepsilon^{2}}\left\|D^{m}(\tilde{v}-v)\right\|^{4}+\frac{3 \varepsilon^{2}\left\|D^{m} \delta\right\|^{2}}{16}\right)
\end{aligned}
$$




$$
\left(I_{4}^{\prime}, \delta\right) \leq 2 c_{7}^{\prime}\left(\frac{4\left\|D^{m} \omega\right\|^{2}}{\varepsilon^{2}}+\frac{4\left\|D^{m} \gamma\right\|^{2}}{\varepsilon^{2}}+\frac{\varepsilon^{2}\left\|D^{m} \delta\right\|^{2}}{8}\right),
$$

which means that

$$
\left|\left(J_{1}^{\prime}, \delta\right)\right| \leq \frac{c_{8}^{\prime}}{\varepsilon^{2}}\left(\left\|D^{m}(\tilde{u}-u)\right\|^{4}+\left\|D^{m}(\tilde{v}-v)\right\|^{4}+\left\|D^{m} \omega\right\|^{2}+\left\|D^{m} \gamma\right\|^{2}\right)+c_{9}^{\prime} \varepsilon^{2}\left\|D^{m} \delta\right\|^{2} .
$$

Taking (3.15)-(3.18) into (3.14), we have

$$
\begin{aligned}
& \frac{1}{2} \frac{\mathrm{d}}{\mathrm{d} t}\left\{\|\theta\|^{2}+\|\delta\|^{2}+\varepsilon^{2}\left(\|\omega\|^{2}+\|\gamma\|^{2}\right)+\left(m^{*}-\beta \varepsilon\right)\left(\left\|A^{\frac{m}{2}} \omega\right\|^{2}+\left\|A^{\frac{m}{2}} \gamma\right\|^{2}\right)\right\} \\
& +\left(\frac{\beta}{2} \lambda_{1}^{m}-\varepsilon\right)\left(\|\theta\|^{2}+\|\delta\|^{2}\right)+\varepsilon^{3}\left(\|\omega\|^{2}+\|\gamma\|^{2}\right)+\varepsilon\left(m_{0}-\beta \varepsilon\right)\left(\left\|A^{\frac{m}{2}} \omega\right\|^{2}+\left\|A^{\frac{m}{2}} \gamma\right\|^{2}\right) \\
& \leq c_{11}\left(\left\|A^{\frac{m}{2}} \omega\right\|^{2}+\left\|A^{\frac{m}{2}} \gamma\right\|^{2}+\|\theta\|^{2}+\|\delta\|^{2}\right)+\frac{c_{11}^{\prime}}{\varepsilon^{2}}\left(\left\|D^{m}(u-u)\right\|^{4}+\left\|D^{m}(v-v)\right\|^{4}\right) \\
& +c_{10}\left(\left\|D^{m}(u-u)\right\|^{2\left(\kappa_{1}+1\right)}+\left\|D^{m}(v-v)\right\|^{2\left(\kappa_{1}+1\right)}\right) \\
& +c_{10}^{\prime}\left(\left\|D^{m}(u-u)\right\|^{2\left(\kappa_{2}+1\right)}+\left\|D^{m}(v-v)\right\|^{2\left(\kappa_{2}+1\right)}\right) .
\end{aligned}
$$

Let $k^{\prime}=\min \left\{\beta \lambda_{1}^{m}-2 \varepsilon, 2 \varepsilon, \frac{2 \varepsilon\left(m_{0}-\beta \varepsilon\right)}{m^{*}-\beta \varepsilon}\right\}>0$,

$$
\begin{aligned}
& \frac{\mathrm{d}}{\mathrm{d} t}\left\{\|\theta\|^{2}+\|\delta\|^{2}+\|\omega\|^{2}+\|\gamma\|^{2}+\left\|A^{\frac{m}{2}} \omega\right\|^{2}+\left\|A^{\frac{m}{2}} \gamma\right\|^{2}\right\} \\
& +k^{\prime}\left(\|\theta\|^{2}+\|\delta\|^{2}+\|\omega\|^{2}+\|\gamma\|^{2}+\left\|A^{\frac{m}{2}} \omega\right\|^{2}+\left\|A^{\frac{m}{2}} \gamma\right\|^{2}\right) \\
& \leq c_{11}\left(\left\|A^{\frac{m}{2}} \omega\right\|^{2}+\|\theta\|^{2}+\left\|A^{\frac{m}{2}} \gamma\right\|^{2}+\|\delta\|^{2}\right)+\frac{c_{11}^{\prime}}{\varepsilon^{2}}\left(\left\|D^{m}(u-u)\right\|^{4}+\left\|D^{m}(v-v)\right\|^{4}\right) \\
& +c_{10}\left(\left\|D^{m}(u-u)\right\|^{2\left(\kappa_{1}+1\right)}+\left\|D^{m}(v-v)\right\|^{2\left(\kappa_{1}+1\right)}\right) \\
& +c_{10}^{\prime}\left(\left\|D^{m}(u-u)\right\|^{2\left(\kappa_{2}+1\right)}+\left\|D^{m}(v-v)\right\|^{2\left(\kappa_{2}+1\right)}\right),
\end{aligned}
$$

applying the Gronwall inequality and (3.6) we deduce from (3.20) that

$$
\begin{aligned}
& \left\{\left\|A^{\frac{m}{2}} \omega\right\|^{2}+\|\theta\|^{2}+\left\|A^{\frac{m}{2}} \gamma\right\|^{2}+\|\delta\|^{2}\right\} \\
& \leq \frac{c_{12}^{\prime}}{c_{11}} \exp \left(c_{11} t\right) \int_{0}^{t}\left(\left\|D^{m}(u-u)\right\|^{4}+\left\|D^{m}(v-v)\right\|^{4}+\left\|D^{m}(u-u)\right\|^{2\left(\kappa_{1}+1\right)}\right. \\
& \left.+\left\|D^{m}(v-v)\right\|^{2\left(\kappa_{1}+1\right)}+\left\|D^{m}(u-u)\right\|^{2\left(\kappa_{2}+1\right)}+\left\|D^{m}(v-v)\right\|^{2\left(\kappa_{2}+1\right)}\right) \mathrm{d} \tau \\
& \leq c_{13}^{\prime} \exp \left(c_{12} t\right)\left(\left\{\|(\xi, \zeta, \eta, \sigma)\|_{E_{0}}^{2}\right\}^{2}+\left\{\|(\xi, \zeta, \eta, \sigma)\|_{E_{0}}^{2}\right\}^{\kappa_{1}+1}+\left\{\|(\xi, \zeta, \eta, \sigma)\|_{E_{0}}^{2}\right\}^{\kappa_{2}+1}\right) .
\end{aligned}
$$


This is equivalent to

$$
\begin{aligned}
& \left\|S(t) \tilde{\rho}_{0}-S(t) \rho_{0}-\left(D S(t) \rho_{0}\right)(\xi, \zeta, \eta, \sigma)\right\|_{E_{0}}^{2} \\
& \leq c_{13}^{\prime} \exp \left(c_{12} t\right)\left(\|(\xi, \zeta, \eta, \sigma)\|_{E_{0}}^{4}+\|(\xi, \zeta, \eta, \sigma)\|_{E_{0}}^{2\left(\kappa_{1}+1\right)}+\|(\xi, \zeta, \eta, \sigma)\|_{E_{0}}^{2\left(\kappa_{2}+1\right)}\right),
\end{aligned}
$$

and consequently $\frac{\left\|S(t) \tilde{\rho}_{0}-S(t) \rho_{0}-\left(D S(t) \rho_{0}\right)(\xi, \zeta, \eta, \sigma)\right\|_{E_{0}}^{2}}{\|(\xi, \zeta, \eta, \sigma)\|_{E_{0}}^{2}} \rightarrow 0$ as $(\xi, \zeta, \eta, \sigma) \rightarrow 0$ in $E_{0}$.

The differentiability of $S(t)$ is proved.

The next step will be used in demonstrating the process of dimension estimation. It seems obvious that the equations (1.1)-(1.2) also can be written as

$$
\begin{gathered}
\varphi_{t}+H(\varphi)=F(\varphi), \\
\qquad(\varphi)=\left(\begin{array}{c}
A^{m} u-\varepsilon\left(\beta A^{m}-\varepsilon\right) u+\left(\beta A^{m}-\varepsilon\right) p \\
\varepsilon v-q \\
A^{m} v-\varepsilon\left(\beta A^{m}-\varepsilon\right) v+\left(\beta A^{m}-\varepsilon\right) q
\end{array}\right), \\
F(\varphi)=\left(\begin{array}{c}
0 \\
f_{1}(x)-g_{1}(u, v)+\left(\begin{array}{c}
1-M\left(\left\|A^{\frac{m}{2}} u\right\|^{2}+\left\|A^{\frac{m}{2}} v\right\|^{2}\right. \\
0
\end{array}\right) A^{m} u \\
f_{2}(x)-g_{2}(u, v)+\left(\begin{array}{c}
1-M \\
A^{\frac{m}{2}} u\left\|^{2}+\right\| A^{\frac{m}{2}} v \|^{2}
\end{array}\right)
\end{array}\right) . A^{m} v
\end{gathered}
$$

Lemma 3.4. For any $\varphi=\left(u_{1}, p_{1}, v_{1}, q_{1}\right)^{\mathrm{T}} \in E_{0}$, we have

$$
(H(\varphi), \varphi) \geq \frac{\varepsilon}{2}\|\varphi\|_{E_{0}}^{2}+\frac{\beta}{4}\left(\left\|A^{\frac{m}{2}} p\right\|^{2}+\left\|A^{\frac{m}{2}} q\right\|^{2}\right)
$$

Proof. For any $\varphi=\left(u_{1}, p_{1}, v_{1}, q_{1}\right)^{\mathrm{T}} \in E_{0}$, through the above definition, we get

$$
\begin{aligned}
(H(\varphi), \varphi)_{E_{0}}= & \left(\varepsilon A^{\frac{m}{2}} u-A^{\frac{m}{2}} p, A^{\frac{m}{2}} u\right)+\left(\varepsilon A^{\frac{m}{2}} v-A^{\frac{m}{2}} q, A^{\frac{m}{2}} v\right) \\
& +\left(A^{m} u-\varepsilon\left(\beta A^{m}-\varepsilon\right) u+\left(\beta A^{m}-\varepsilon\right) p, p\right) \\
& +\left(A^{m} v-\varepsilon\left(\beta A^{m}-\varepsilon\right) v+\left(\beta A^{m}-\varepsilon\right) q, q\right) \\
= & \varepsilon\left(\left\|A^{\frac{m}{2}} u\right\|^{2}+\left\|A^{\frac{m}{2}} v\right\|^{2}\right)+\beta\left(\left\|A^{\frac{m}{2}} p\right\|^{2}+\left\|A^{\frac{m}{2}} q\right\|^{2}\right)-\varepsilon\left(\|p\|^{2}+\|q\|^{2}\right) \\
& -\beta \varepsilon\left(\left(A^{m} u, p\right)+\left(A^{m} v, q\right)\right)+\varepsilon^{2}((u, p)+(v, q)) .
\end{aligned}
$$

By applying the Holder inequality, Young's inequality and Poincare inequality, we deal with the terms in (3.26) by as follows 


$$
\begin{aligned}
& -\beta \varepsilon\left(\left(A^{m} u, p\right)+\left(A^{m} v, q\right)\right) \geq-\frac{\beta^{2} \varepsilon^{2}}{2}\left(\left\|A^{\frac{m}{2}} u\right\|^{2}+\left\|A^{\frac{m}{2}} v\right\|^{2}+\left\|A^{\frac{m}{2}} p\right\|^{2}+\left\|A^{\frac{m}{2}} q\right\|^{2}\right), \quad \text { (3.27) } \\
& \qquad \varepsilon^{2}((u, p)+(v, q)) \geq-\frac{\varepsilon^{2}}{2 \lambda_{1}^{m}}\left(\left\|A^{\frac{m}{2}} u\right\|^{2}+\left\|A^{\frac{m}{2}} v\right\|^{2}\right)-\frac{\varepsilon^{2}}{2}\left(\|p\|^{2}+\|q\|^{2}\right), \\
& \text { with } 0<\varepsilon<\min \left\{\sqrt{\frac{3}{2 \beta}}, \frac{\lambda_{1}^{m}}{1+\beta^{2} \lambda_{1}^{m}}, \frac{-3+\sqrt{9+6\left(1+\beta^{2} \lambda_{1}^{m}\right) \beta \lambda_{1}^{m}}}{2\left(1+\beta^{2} \lambda_{1}^{m}\right)}\right\}, \text { and substituting }
\end{aligned}
$$
(3.27)-(3.28) into (3.26), we obtain

$$
\begin{aligned}
(H(\varphi), \varphi) \geq & \left(\varepsilon-\frac{\beta^{2} \varepsilon^{2}}{2}-\frac{\varepsilon^{2}}{2 \lambda_{1}^{m}}\right)\left(\left\|A^{\frac{m}{2}} u\right\|^{2}+\left\|A^{\frac{m}{2}} v\right\|^{2}\right) \\
& +\left(\beta-\frac{\beta^{2} \varepsilon^{2}}{2}\right)\left(\left\|A^{\frac{m}{2}} p\right\|^{2}+\left\|A^{\frac{m}{2}} q\right\|^{2}\right)+\left(-\varepsilon-\frac{\varepsilon^{2}}{2}\right)\left(\|p\|^{2}+\|q\|^{2}\right) \\
\geq & \frac{\varepsilon}{2}\left(\left\|A^{\frac{m}{2}} u\right\|^{2}+\left\|A^{\frac{m}{2}} v\right\|^{2}+\|p\|^{2}+\|q\|^{2}\right)+\frac{\beta}{4}\left(\left\|A^{\frac{m}{2}} p\right\|^{2}+\left\|A^{\frac{m}{2}} q\right\|^{2}\right) \\
\geq & \frac{\varepsilon}{2}\|\varphi\|_{E_{0}}^{2}+\frac{\beta}{4}\left(\left\|A^{\frac{m}{2}} p\right\|^{2}+\left\|A^{\frac{m}{2}} q\right\|^{2}\right) .
\end{aligned}
$$

The proof of lemma 3.4 is completed.

Consider the first variation equation of (3.23)

$$
\Psi^{\prime}+P(\varphi) \Psi=\Gamma_{1}(\varphi) \Psi+\Gamma_{2}(\varphi) \Psi
$$

where $\Psi=(U, P, V, Q)^{\mathrm{T}} \in E_{0}, \quad P=U_{t}+\varepsilon U, Q=V_{t}+\varepsilon V$ and $\varphi=(u, p, v, q)^{\mathrm{T}} \in E_{0}$ is a solution of (3.23), $\Psi(0)=\{\xi, \zeta, \eta, \sigma\} \in E_{0}, t>0$.

$$
\begin{gathered}
P(\varphi)=\left(\begin{array}{cccc}
\varepsilon I & -I & 0 & 0 \\
(1-\beta \varepsilon) A^{m}+\varepsilon^{2} I & \beta A^{m}-\varepsilon I & 0 & 0 \\
0 & 0 & \varepsilon I & -I \\
0 & 0 & (1-\beta \varepsilon) A^{m}+\varepsilon^{2} I & \beta A^{m}-\varepsilon I
\end{array}\right), \\
\Gamma_{1}(\varphi)=\left(\begin{array}{cccc}
0 & 0 & 0 & 0 \\
-g_{1 u}(u, v) & 0 & -g_{1 v}(u, v) & 0 \\
0 & 0 & 0 & 0 \\
-g_{2 u}(u, v) & 0 & -g_{2 v}(u, v) & 0
\end{array}\right)
\end{gathered}
$$

$\Gamma_{2}(\varphi)$

$$
=\left(\begin{array}{c}
0 \\
(1-M(s)) A^{m} U-2 M^{\prime}(s)\left(A^{\frac{m}{2}} U, A^{\frac{m}{2}} u\right) A^{m} u-2 M^{\prime}(s)\left(A^{\frac{m}{2}} V, A^{\frac{m}{2}} v\right) A^{m} u \\
0 \\
(1-M(s)) A^{m} V-2 M^{\prime}(s)\left(A^{\frac{m}{2}} U, A^{\frac{m}{2}} u\right) A^{m} v-2 M^{\prime}(s)\left(A^{\frac{m}{2}} V, A^{\frac{m}{2}} v\right) A^{m} v
\end{array}\right) .
$$


Lemma 3.5. ([2]) Let there be given $V, H$, and $A$ as above. Then for any $s, 0 \leq s<1$, and for any orthonormal family of elements of $V \times H$, $\left\{\xi_{i}, \zeta_{i}\right\}, i=1, \cdots, m$, we have

$$
\sum_{i}^{m}\left|A^{s / 2} \xi_{i}\right|^{2} \leq \sum_{i}^{m} \lambda_{i}^{s-1}
$$

where $\left\{\lambda_{i}\right\}_{i \in N}$ is the eigenvalue of $A^{m}$.

Proof. This is a direct consequence for Lemma 6.3 of [2].

Theorem 3.1. Let $A$ be the global attractor of problem (1.1)-(1.5), then the Hausdorff dimension of global attractor $A$ is less than or equal to $n_{0}$ and its fractal dimension is less than or equal to $2 n_{0}$.

$$
\begin{gathered}
d_{H}(A) \leq \min \left\{n_{0} \mid n_{0} \in N, \frac{1}{n_{0}} \sum_{j=1}^{n_{0}} \lambda_{j}^{v-1}<\frac{\beta \varepsilon}{16 k^{2}}\right\}, \\
v=\left\{\begin{array}{l}
\frac{(n-2 m)(r-1)-2 m}{2 m}, \frac{n}{n-2 m} \leq r \leq \frac{n+2 m}{n-2 m}, n \geq 2 m, \\
0, \quad n<2 m \text { or } 0 \leq r \leq \frac{n}{n-2 m}, n \geq 2 m .
\end{array} \text { (or } r^{\prime}\right)
\end{gathered}
$$

Proof. Let $n_{0} \in N$ be settled. Consider $n_{0}$ solutions $\Psi_{1}, \Psi_{2}, \cdots, \Psi_{n_{0}}$ of (3.30), and we memorize that

$$
\begin{aligned}
& \left|\Psi_{1}(t) \wedge \cdots \wedge \Psi_{n_{0}}(t)\right|_{\wedge{ }^{n_{0}} E_{0}} \\
& =\left|\Psi_{1}(0) \wedge \cdots \wedge \Psi_{n_{0}}(0)\right|_{\wedge{ }^{n_{0}} E_{0}} \exp \int_{0}^{t} \operatorname{Tr} F^{\prime}(\varphi(\tau)) \circ Q_{n_{0}}(\tau) \mathrm{d} \tau,
\end{aligned}
$$

we see that $\varphi(\tau)=(u(\tau), p(\tau), v(\tau), q(\tau))$, $Q_{n_{0}}(\tau)=Q_{n_{0}}\left(\tau, \varphi_{0} ; \Psi_{1}(0), \cdots, \Psi_{n_{0}}(0)\right)$ is the orthogonal projection in $E_{0}$ onto the space spanned by $\Psi_{1}(\tau), \cdots, \Psi_{m}(\tau)$. At a given time $\tau$, let $h_{j}(\tau)=\left\{\xi_{j}, \zeta_{j}, \eta_{j}, \sigma_{j}\right\}, j=1, \cdots, n_{0}$, denote an orthonormal basis of

$$
\begin{gathered}
Q_{n_{0}}(\tau) E_{0}=\operatorname{Span}\left\{\Psi_{1}(\tau), \Psi_{2}(\tau), \cdots, \Psi_{n_{0}}(\tau)\right\}, \\
\operatorname{Tr} F^{\prime}(\varphi(\tau)) \circ Q_{n_{0}}(\tau)=\sum_{j=1}^{+\infty}\left(\Gamma^{\prime}(\varphi(\tau)) \circ Q_{n_{0}}(\tau) h_{j}(\tau), h_{j}(\tau)\right)_{E_{0}} \\
=\sum_{j=1}^{n_{0}}\left(\Gamma^{\prime}(\varphi(\tau)) h_{j}(\tau), h_{j}(\tau)\right)_{E_{0}} .
\end{gathered}
$$

With respect to the scalar product $(\cdot, \cdot)_{E_{0}}$ and norm $\|\cdot\|_{E_{0}}$, we omit for the moment variable $\tau$,

$$
\begin{aligned}
& \left(h_{j}, \tilde{h}_{j}\right)_{E_{0}}=\left(\xi_{j}, \tilde{\xi}_{j}\right)+\left(\zeta_{j}, \tilde{\zeta}_{j}\right)+\left(\eta_{j}, \tilde{\eta}_{j}\right)+\left(\sigma_{j}, \tilde{\sigma}_{j}\right), \\
& \left\|h_{j}\right\|_{E_{0}}^{2}=\left(h_{j}, h_{j}\right)_{E_{0}}=\left\|A^{\frac{m}{2}} \xi_{j}\right\|^{2}+\left\|\zeta_{j}\right\|^{2}+\left\|A^{\frac{m}{2}} \eta_{j}\right\|^{2}+\left\|\sigma_{j}\right\|^{2}=1 .
\end{aligned}
$$

By the Lemma 3.4, we have

$$
-\left(P(\varphi) h_{j}, h_{j}\right) \leq-\frac{\varepsilon}{2}-\frac{\beta}{4}\left(\left\|A^{\frac{m}{2}} \zeta_{j}\right\|^{2}+\left\|A^{\frac{m}{2}} \sigma_{j}\right\|^{2}\right),
$$




$$
\begin{aligned}
\left(\Gamma_{1}(\varphi) h_{j}, h_{j}\right)_{E_{0}}= & \left(-g_{1 u}(u, v) \xi_{j}, \zeta_{j}\right)+\left(-g_{1 v}(u, v) \eta_{j}, \zeta_{j}\right) \\
& +\left(-g_{2 u}(u, v) \xi_{j}, \sigma_{j}\right)+\left(-g_{2 v}(u, v) \eta_{j}, \sigma_{j}\right) \\
& \leq\left\|A^{-\frac{m}{2}} g_{1 u}(u, v) \xi_{j}\right\|\left\|A^{\frac{m}{2}} \zeta_{j}\right\|+\left\|A^{-\frac{m}{2}} g_{1 v}(u, v) \eta_{j}\right\|\left\|A^{\frac{m}{2}} \zeta_{j}\right\| \\
& \leq\left\|A^{-\frac{m}{2}} g_{2 u}(u, v) \xi_{j}\right\|\left\|A^{\frac{m}{2}} \sigma_{j}\right\|+\left\|A^{-\frac{m}{2}} g_{2 v}(u, v) \eta_{j}\right\| A^{\frac{m}{2}} \sigma_{j} \| .
\end{aligned}
$$

By the assumption (H3) in [1], the mean value theorem and the Sobolev embedding theorem

$$
H_{0}^{m s}(\Omega) \subset D\left(A^{\frac{m s}{2}}\right) \subset H^{m s}(\Omega) \subset L^{q}(\Omega) \subset L^{2}(\Omega) \subset L^{q^{\prime}}(\Omega) \subset H^{m s}(\Omega)
$$

where

$$
\frac{1}{q}=\frac{1}{2}-\frac{m s}{n}, \frac{1}{q}+\frac{1}{q^{\prime}}=1, m s \in[0,1]
$$

For $n=1, H_{0}^{m}(\Omega) \subset L^{\infty}(\Omega) \subset L^{1}(\Omega) \subset H^{-m}(\Omega) \subset\left(H_{0}^{m}(\Omega)\right)^{\prime}$, we can easily obtain that

$$
\begin{aligned}
& \left\|A^{-\frac{m}{2}} g_{1 u}(u, v) \xi_{j}\right\| \leq c\left\|g_{1 u}(u, v) \xi_{j}\right\|_{L^{1}} \leq c_{13}\left(R_{0}\right)\left\|\xi_{j}\right\|, \\
& \left\|A^{-\frac{m}{2}} g_{1 v}(u, v) \eta_{j}\right\| \leq c\left\|g_{1 v}(u, v) \eta_{j}\right\|_{L^{1}} \leq c_{14}\left(R_{0}\right)\left\|\eta_{j}\right\|, \\
& \left\|A^{-\frac{m}{2}} g_{2 u}(u, v) \xi_{j}\right\| \leq c\left\|g_{2 u}(u, v) \xi_{j}\right\|_{L^{1}} \leq c_{15}\left(R_{0}\right)\left\|\xi_{j}\right\|, \\
& \left\|A^{-\frac{m}{2}} g_{2 v}(u, v) \eta_{j}\right\| \leq c\left\|g_{2 v}(u, v) \eta_{j}\right\|_{L^{1}} \leq c_{16}\left(R_{0}\right)\left\|\eta_{j}\right\| .
\end{aligned}
$$

For $1<n<2 m, H_{0}^{m}(\Omega) \subset L^{q}(\Omega) \subset H^{-m}(\Omega) \subset\left(H_{0}^{m}(\Omega)\right)^{\prime}, q>0$, we have

$$
\begin{aligned}
& \left\|A^{-\frac{m}{2}} g_{1 u}(u, v) \xi_{j}\right\| \leq c\left\|g_{1 u}(u, v) \xi_{j}\right\|_{L^{2}} \leq c_{17}\left(R_{0}\right)\left\|\xi_{j}\right\|, \\
& \left\|A^{-\frac{m}{2}} g_{1 v}(u, v) \eta_{j}\right\| \leq c\left\|g_{1 v}(u, v) \eta_{j}\right\|_{L^{\frac{2}{2}}} \leq c_{18}\left(R_{0}\right)\left\|\eta_{j}\right\|, \\
& \left\|A^{-\frac{m}{2}} g_{2 u}(u, v) \xi_{j}\right\| \leq c\left\|g_{2 u}(u, v) \xi_{j}\right\|_{L^{\frac{2}{2}}} \leq c_{19}\left(R_{0}\right)\left\|\xi_{j}\right\|, \\
& \left\|A^{-\frac{m}{2}} g_{2 v}(u, v) \eta_{j}\right\| \leq c\left\|g_{2 v}(u, v) \eta_{j}\right\|_{L^{\frac{2}{2}}} \leq c_{20}\left(R_{0}\right)\left\|\eta_{j}\right\| .
\end{aligned}
$$

For $n \geq 2 m$, there exist $c_{21}\left(R_{0}\right), c_{22}\left(R_{0}\right), c_{23}\left(R_{0}\right), c_{24}\left(R_{0}\right)>0$, such that

$$
\left\|A^{-\frac{m}{2}} g_{1 u}(u, v) \xi_{j}\right\| \leq c\left\|g_{1 u}(u, v) \xi_{j}\right\|_{L^{n+2 m}} \leq c_{21}\left(R_{0}\right)\left\|A^{\frac{m}{2} v} \xi_{j}\right\|,
$$




$$
\begin{aligned}
& \left\|A^{-\frac{m}{2}} g_{1 v}(u, v) \eta_{j}\right\| \leq c\left\|g_{1 v}(u, v) \eta_{j}\right\|_{L^{n+2 m}} \leq c_{22}\left(R_{0}\right)\left\|A^{\frac{m}{2} v} \eta_{j}\right\|, \\
& \left\|A^{-\frac{m}{2}} g_{2 u}(u, v) \xi_{j}\right\| \leq c\left\|g_{2 u}(u, v) \xi_{j}\right\|_{L^{\frac{2 n}{n+2 m}}} \leq c_{23}\left(R_{0}\right)\left\|A^{\frac{m}{2} v} \xi_{j}\right\|, \\
& \left\|A^{-\frac{m}{2}} g_{2 v}(u, v) \eta_{j}\right\| \leq c\left\|g_{2 v}(u, v) \eta_{j}\right\|_{L^{n+2 m}} \leq c_{24}\left(R_{0}\right)\left\|A^{\frac{m}{2} v} \eta_{j}\right\|
\end{aligned}
$$

where $v$ is as in (3.36), then setting $k=2 \max \left\{c_{i}\left(R_{0}\right), i=13 \sim 24\right\}$

$$
\begin{aligned}
& \left(\Gamma_{1}(\varphi) h_{j}, h_{j}\right)_{E_{0}} \leq \frac{k}{2}\left(\left\|A^{\frac{m}{2} v} \xi_{j}\right\|+\left\|A^{\frac{m}{2} v} \eta_{j}\right\|\right)\left(\left\|A^{\frac{m}{2}} \zeta_{j}\right\|+\left\|A^{\frac{m}{2}} \sigma_{j}\right\|\right), \\
& \left(\Gamma_{2}(\varphi) h_{j}, h_{j}\right)_{E_{0}} \\
= & (1-M(s))\left(A^{m} \xi_{j}, \zeta_{j}\right)-2 M^{\prime}(s)\left(A^{\frac{m}{2}} \xi_{j}, A^{\frac{m}{2}} u\right)\left(A^{m} u, \zeta_{j}\right) \\
& -2 M^{\prime}(s)\left(A^{\frac{m}{2}} \eta_{j}, A^{\frac{m}{2}} v\right)\left(A^{m} u, \zeta_{j}\right)+(1-M(s))\left(A^{m} \eta_{j}, \sigma_{j}\right) \\
& -2 M^{\prime}(s)\left(A^{\frac{m}{2}} \xi_{j}, A^{\frac{m}{2}} u\right)\left(A^{m} v, \sigma_{j}\right)-2 M^{\prime}(s)\left(A^{\frac{m}{2}} \eta_{j}, A^{\frac{m}{2}} v\right)\left(A^{m} v, \sigma_{j}\right) \\
\leq & \left.\left(1-m_{0}\right) \lambda_{j}^{\frac{m}{2}}\left(\left\|A^{\frac{m}{2}} \xi_{j}\right\|\left\|\zeta_{j}\right\|+\left\|A^{\frac{m}{2}} \eta_{j}\right\|\left\|\sigma_{j}\right\|\right)^{2}\right) \\
& +2 c_{25}\left(\left\|A^{\frac{m}{2}} \xi_{j}\right\| \zeta_{j}\|+\| A^{\frac{m}{2}} \eta_{j}\|\| \zeta_{j} \|\right)+2 c_{25}\left(\left\|A^{\frac{m}{2}} \xi_{j}\right\|\left\|\sigma_{j}\right\|+\left\|A^{\frac{m}{2}} \eta_{j}\right\| \sigma_{j} \|\right) \\
\leq & \frac{\left(1-m_{0}\right)}{2} \lambda_{j}^{\frac{m}{2}}\left(\left\|A^{\frac{m}{2}} \xi_{j}\right\|^{2}+\left\|\zeta_{j}\right\|^{2}+\left\|A^{\frac{m}{2}} \eta_{j}\right\|^{2}+\left\|\sigma_{j}\right\|^{2}\right) \\
& +2 c_{25}\left(\left\|A^{\frac{m}{2}} \xi_{j}\right\|^{2}+\left\|\zeta_{j}\right\|^{2}+\left\|A^{\frac{m}{2}} \eta_{j}\right\|+\left\|\zeta_{j}\right\|^{2}\right) \\
\leq & \frac{\left(1-m_{0}\right)}{2} \lambda_{j}^{\frac{m}{2}}+2 c_{25},
\end{aligned}
$$

let $0<\alpha=\left(\left\|\zeta_{j}\right\|^{2}+\left\|\sigma_{j}\right\|^{2}\right)<1$

$$
\begin{aligned}
& \left(\Gamma^{\prime}(\varphi) h_{j}, h_{j}\right)_{E_{0}} \\
& =\left(\left(-P(\varphi)+\Gamma_{1}(\varphi)+\Gamma_{2}(\varphi)\right) h_{j}, h_{j}\right) \\
& \leq\left(-\frac{\varepsilon}{2}-\frac{\alpha \beta}{8} \lambda_{j}^{m}+\frac{\left(1-m_{0}\right)}{2} \lambda_{j}^{\frac{m}{2}}+2 c_{25}\right)+\frac{2 k^{2}}{\beta}\left(\left\|A^{\frac{m}{2} v} \xi_{j}\right\|^{2}+\left\|A^{\frac{m}{2} v} \eta_{j}\right\|^{2}\right) . \\
\text { If } & -\frac{\alpha \beta}{8} \lambda_{j}^{m}+\frac{\left(1-m_{0}\right)}{2} \lambda_{j}^{\frac{m}{2}}<\frac{\varepsilon}{4},
\end{aligned}
$$




$$
\begin{aligned}
\sum_{j=1}^{n_{0}}\left(\Gamma^{\prime}(\varphi) h_{j}, h_{j}\right)_{E_{0}} & =\sum_{j=1}^{n_{0}}\left(\left(-P(\varphi)+\Gamma_{1}(\varphi)+\Gamma_{2}(\varphi)\right) h_{j}, h_{j}\right) \\
& =-\frac{\varepsilon}{4} n_{0}+\frac{4 k^{2}}{\beta} \sum_{j}^{n_{0}} \lambda_{j}^{\nu-1},
\end{aligned}
$$

and if $\frac{1}{n_{0}} \sum_{j=1}^{n_{0}} \lambda_{j}^{\nu-1}<\frac{\beta \varepsilon}{16 k^{2}}$

$$
\begin{gathered}
q_{n_{0}}(t)=\sup _{\substack{\varphi_{0} \in \mathcal{A} \Psi_{j}(0) \in E_{0} \\
\left\|\Psi_{j}(0)\right\|_{E_{0}} \leq 1 \\
j=1, \cdots, n_{0}}}\left\{\frac{1}{t} \int_{0}^{t} \operatorname{Tr} F^{\prime}(\varphi(\tau)) \circ Q_{n_{0}}(\tau) \mathrm{d} \tau\right\} \\
\leq-\frac{k^{2} n_{0}}{4}\left(\frac{\varepsilon}{k^{2}}-\frac{16}{\beta n_{0}} \sum_{j=1}^{n_{0}} \lambda_{j}^{\nu-1}\right) \\
q_{n_{0}}=\lim _{t \rightarrow \infty} \sup q_{n_{0}}(t)<0 .
\end{gathered}
$$

The proof of theorem 3.1 is completed.

\section{Acknowledgements}

The authors express their sincere thanks to the anonymous referee for his/her careful reading of the paper, giving valuable suggestions and comments, which have greatly improved the presentation of this paper.

\section{References}

[1] Lin, G.G. and Hu, L.J. (2017) The Attractor for a Class of Higher-Order Coupled Kirchhoff Type Equations with Strong Linear Damping. European Journal of Mathematics and Computer Science, 4, 63-77.

[2] Teman, R. (1988) Infinite-Dimensional Dynamical Systems in Mechanics and Physics. Springer, New York. https://doi.org/10.1007/978-1-4684-0313-8

[3] Huang, Y. (1998) Estimates of the Hausdorff Dimension and Fractal Dimension of Global Attractors for Nonlinear Wave Equations with Damping. Acta Mathematics Applicatae Sinica, 21, 257-266.

[4] Zhou, S. (1999) On Dimension of Global Attractor for Damped Nonlinear Wave Equations. Proceedings of the American Mathematical Society, 40, 1432-1438.

[5] Zhou, S.F. (2000) Attractor and Dimension for Strongly Damped Nonlinear Wave Equation. Acta Mathematicae Applicatea Sinica, 16, 266-273. https://doi.org/10.1007/BF02679891

[6] Yang, Z.J. (2007) Longtime Behavior of the Kirchhoff Type Equation with Strong Damping on. Journal of Differential Equations, 242, 269-286. https://doi.org/10.1016/j.jde.2007.08.004

[7] Yang, Z.J. (2010) Global Attractors and Their Hausdorff Dimensions for a Class of Kirchhoff Models. Journal of Mathematical Physics, 51, 231.

[8] Fan, X.M. and Zhou, S.F. (2004) Kernel Sections for Non-Autonomous Strongly Damped Wave Equations of Non-Degenerate Kifchhoff-Type. Applied Mathematics and Computation, 158, 253-266. https://doi.org/10.1016/j.amc.2003.08.147

[9] Lin, G.G. and Gao, Y.L. (2017) The Global and Exponential Attractors for the HigherOrder Kirchhoff-Type Equation with Strong Linear Damping. Journal of Mathematics Research, 9, 145-167. https://doi.org/10.5539/jmr.v9n4p145 